\title{
Introduction to the Special Issue on Tissue Engineering and Regenerative Medicine
}

Welcome to this special issue of the Annals of Biomedical Engineering with a focus on Tissue Engineering and Regenerative Medicine. In this issue, we collected 18 research articles and reviews on this topic. These manuscripts cover three important areas: stem cell engineering and microenvironmental regulation, biomaterial design for tissue engineering, and in vivo tissue repair and regeneration. Recent advances in stem cell biology have made it possible to use in vitro primed or undifferentiated adult stem cells, as well as differentiated cells derived from pluripotent or adult stem cells as a source for cell transplantation in vivo and studies of development and disease in vitro. Specifically, the cell reprogramming technologies allow the efficient generation of various human cell types (e.g. neurons, cardiomyocytes, pancreatic cells) otherwise unattainable by other methods. However, to harness the full potential of stem cells in tissue engineering and regenerative medicine, significant amount of work is still needed to better understand the role of microenvironmental factors in cell expansion and differentiation in vitro, biomaterial properties leading to mature tissue structure and function, host-graft interactions resulting in enhanced implant survival and integration, and conditions that would allow successful tissue engineering in situ.

The first section of the Special Issue will thus highlight the strategies to obtain large quantities of clinical grade pluripotent stem cells and provide examples of the use of bone marrow-derived mesenchymal stem cells in both soft and hard tissue engineering. Differentiation and functional properties of stem cells can be significantly improved by application of appropriate biophysical stimulation and specific geometrical, microstructural, and biochemical cues. Examples of these strategies are discussed as they relate to engineering of striated muscles, neuronal differentiation, and mechanistic studies of tissue patterning in development and disease. The second section will focus on the biomaterial scaffolds as a key factor for the successful formation and differentiation of engineered tissues and a vehicle for targeted manipulation of cellular fate via controlled gene and protein release. Specifically, several examples are provided in which biochemical and biophysical properties of biomaterial scaffolds (e.g. composition, micro/nano structure, porosity, degradability) as well as cell seeding conditions were investigated to promote the survival, differentiation, function, and antimicrobial activity of engineered neuronal, vascular, osteogenic, and skin tissues. Finally, the third section of this Special Issue will review previous progress and provide examples of new research in an important area of in vivo tissue engineering. Improved understanding of the relevant mechanisms of in vivo repair and regeneration by use of cells alone, cells and biomaterials, or biomaterials alone will be critical to our ability to translate research findings from bench to bedside. Relevant topics will be discussed as they relate to foreign body response, inflammation, wound healing, biomaterial-or cellbased growth factor delivery, and use of biomaterials for in situ tissue engineering or manipulation of host tissue mechanics.

Overall, we hope that this Special Issue will provide readers with important insights into the recent progress and ongoing challenges in this fast growing and exciting field. We believe that future applications of tissue engineering and regenerative medicine will lead to significant improvements in health of millions people worldwide.

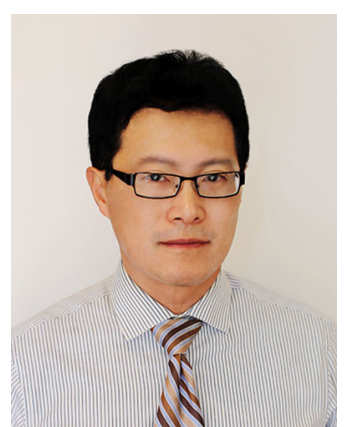

Tzung Hsiai.

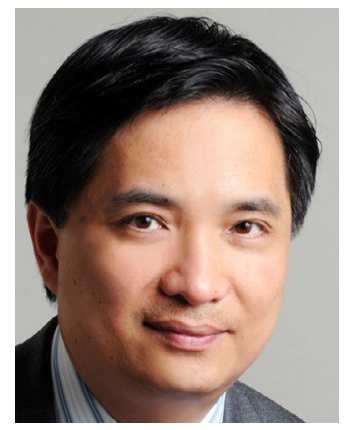

Song Li.

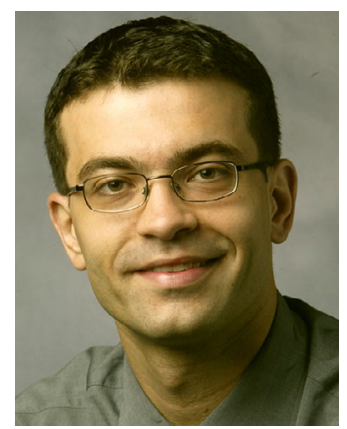

Nenad Bursac. 
TzUng HsiaI

University of California, Los Angeles, Los Angeles,

CA, USA

Electronic mail: THsiai@mednet.ucla.edu

SoNG LI

University of California, Berkeley, Berkeley,

CA, USA
NenAd Bursac

Faculty of Cardiology, Duke University, Durham, NC, USA 